\title{
A monolithically integrated micro fluidic channel in a silicon-based photonic-integrated-circuit technology for biochemical sensing
}

Steglich, Patrick, Paul, Martin, Mai, Christian, Böhme, Andrea, Bondarenko, Siegfried, et al.

Patrick Steglich, Martin Paul, Christian Mai, Andrea Böhme, Siegfried Bondarenko, Michael G. Weller, Andreas Mai, "A monolithically integrated micro fluidic channel in a silicon-based photonic-integrated-circuit technology for biochemical sensing," Proc. SPIE 11772, Optical Sensors 2021, 1177206 (18 April 2021); doi: 10.1117/12.2588791 


\title{
A monolithically integrated microfluidic channel in a silicon-based photonic-integrated-circuit technology for biochemical sensing
}

\author{
Patrick Steglich ${ }^{\mathrm{a}, \mathrm{b}}$, Martin Paul ${ }^{\mathrm{c}}$, Christian Mai ${ }^{\mathrm{a}}$, Andrea Böhme ${ }^{\mathrm{b}}$, Siegfried Bondarenko ${ }^{\mathrm{b}}$, \\ Michael G. Weller ${ }^{\mathrm{c}}$, and Andreas Mai ${ }^{\mathrm{a}, \mathrm{b}}$ \\ ${ }^{a}$ IHP - Leibniz-Institut für innovative Mikroelektronik, 15236 Frankfurt (Oder), Germany \\ ${ }^{b}$ Technische Hochschule Wildau, 15745 Wildau, Germany \\ ${ }^{\mathrm{c}}$ Federal Institute for Materials Research and Testing (BAM), 12489 Berlin, Germany
}

\begin{abstract}
In this work, a cost-effective optofluidic system is propossed and preliminary experimental results are presented. A microfluidic channel monolithically integrated into a photonic integrated circuit technology is used in conjunction with a cyclo-olefin copolymer (COC) substrate to provide fluidic in- and output ports. We report on initial experimental results as well as on the simple and cost-effective fabrication of this optofluidic system by means of micro-milling.
\end{abstract}

Keywords: Biosensors, Biophotonics, Optical sensors, Optoelectronic and photonic sensors, Ring resonators, Silicon photonics

\section{INTRODUCTION}

Silicon photonic biosensors in a photonic integrated circuit (PIC) technology using silicon-on-insulator wafer provide a scalable platform for label-free sensing of various substances ${ }^{1}$ as well as temperature sensing. ${ }^{2}$ Recent advances in the design of sub-wavelength grating waveguides ${ }^{3}$ and coherent phase detection ${ }^{4}$ have led to a high sensitivity and demonstrate their high performance.

Since scalable on-chip light sources are still not available through open-access silicon photonics platforms, ${ }^{5}$ an external light source is required. In order to use an external light source like a tunable laser or a super luminescence diode, the photonic sensor can be integrated in conjunction with grating coupler for an effective fiber-to-chip light coupling. ${ }^{6}$ At the same time, a photodiode can be integrated directly on the same chip. In this case, the photodiode requires electrical connection, which can be realized by wire bonding.

As a consequence, the main challenge of photonic biosensors lies in the monolithic integration of grating coupler, photodiode and microfluidics. All three components are placed on the same side of the chip, occupying a relatively large area, which increases fabrication costs drastically. This leaves the high potential of silicon photonic sensors unleashed.

To overcome this issue, we propose and investigate a cost-effective optofluidic system by integrating monolithically a microfluidic channel into a PIC technology in conjunction with a easy to fabricate microfluidic interconnection, consisting of a cyclo-olefin copolymer (COC) substrate.

Further author information (Patrick Steglich):

steglich@ihp-microelectronics.com

Optical Sensors 2021, edited by Francesco Baldini, Jiri Homola, Robert A. Lieberman, Proc. of SPIE

Vol. 11772, $1177206 \cdot$ C $2021 \mathrm{SPIE} \cdot$ CCC code: 0277-786X/21/\$21 · doi: 10.1117/12.2588791 


\section{EXPERIMENTAL RESULTS}

The fabrication of the photonic biosensor is realized on a $200 \mathrm{~mm}$ silicon-on-insulator technology platform. While the photonic biosensor is structured from the top of the wafer, the microfluidic channel is locally introduced by a backside release process using a combination of dry and wet etching. For the local-backside-etching of the $760 \mu \mathrm{m}$ thick silicon substrate a deep reactive ion etching (DRIE) process in conjunction with a hard mask was used with a very high selectivity of silicon dioxide to silicon (selectivity $\mathrm{SiO} 2 \mathrm{Si}$ of 1:200). This guarantees a very controlled stop on the buried oxide (BOX). We chose a combination of RIE and wet etching to remove the BOX because the waveguide structures could be damaged if only RIE. Drawback of a pure chemical wet etching is an extended process time due to low etch rates of the BOX. Figure 1a shows a fabricated photonic sensor-chip. A comprehensive description of the fabrication process can be found elsewhere. ${ }^{7-9}$

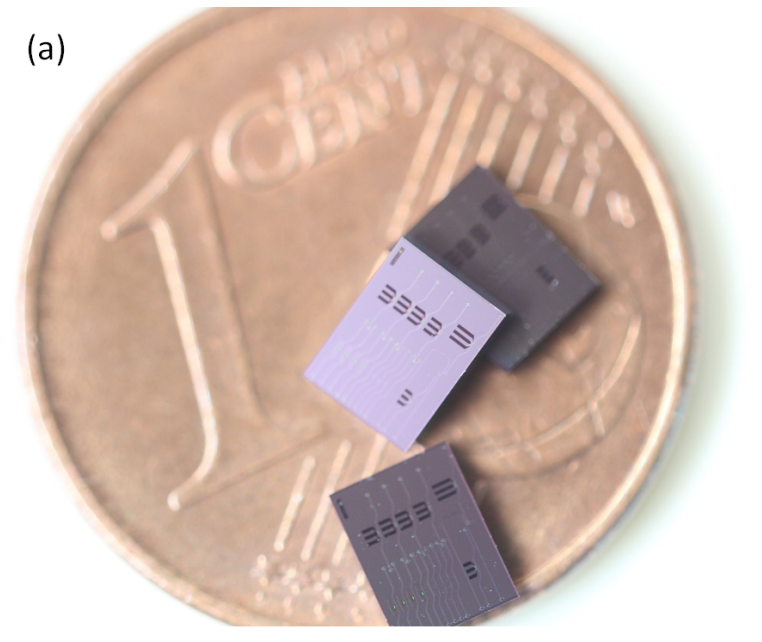

(c)

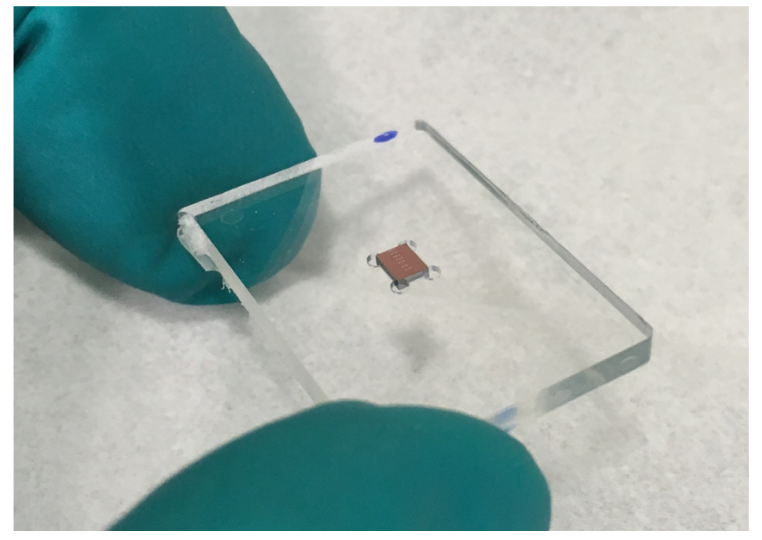

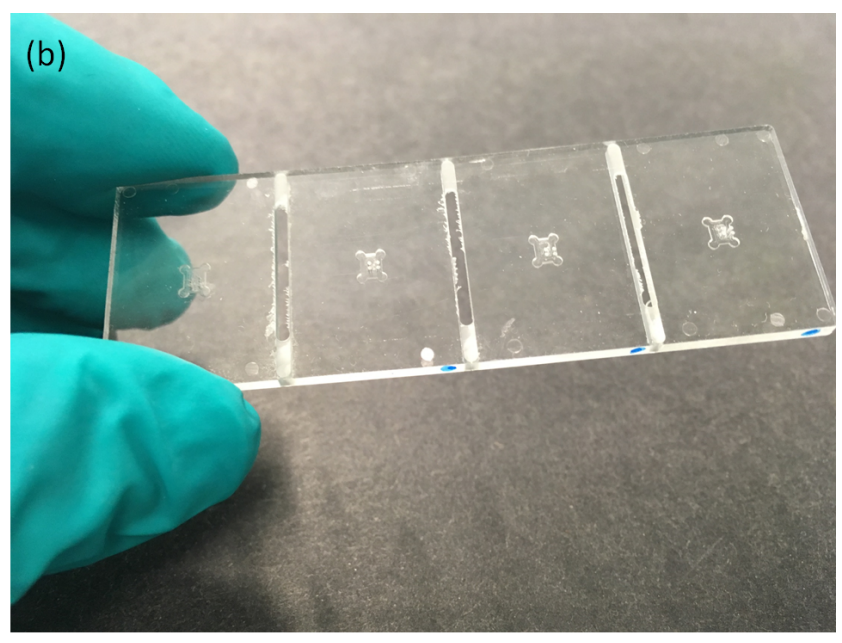

(d)

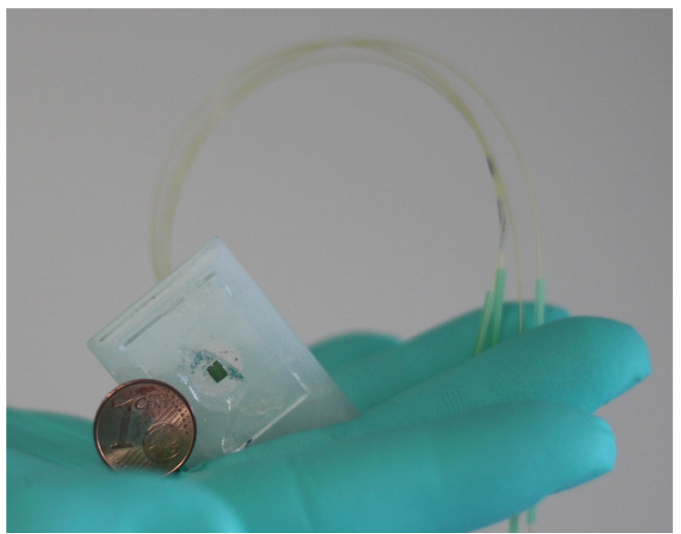

Figure 1. (a) Photonic sensor-chips fabricated in a PIC technology. (c) A row of COC substrates fabricated by means of micro-milling. (d) Photonic sensor-chip packaged with COC substrate and PEEK capillary tubes. (An additional handling substrate is used to be compatible with an existing sample holder.)

Additionally, the presented approach allows a separation of the microfluidic from the optical interconnections. Here, fiber-to-chip light coupling is used on the top side of the sensor chip, and a microfluidic interconnection formed by two holes in a COC substrate is employed on the backside of the chip, where each hole is in conjunction with a polyether ether ketone (PEEK) capillary tube. This microfluidic interconnection as well as a recess for a precise placement of the sensor-chip is realized by means of micro-milling, having a repeatability below $1 \mu \mathrm{m}$. This fabrication enables a mass production and a row of fabricated COC substrates is shown in Figure 1b. Figure 1c shows a single COC substrate with photonic chip, while Figure 1d shows a fully packaged photonic sensor-chip including a carrier substrate. In Figure 2, a schematic view of the COC substrate and a microscopic picture of 
the in- and outlet is shown.

A photonic biosensor based on a silicon micro-ring resonator is employed. In particular, we use a hybridwaveguide ring resonator, consisting of a rib waveguide and a partially on a slot waveguide. To test the viability of the fabricated photonic sensor, we perform a homogeneous sensing experiment to evaluate the sensitivity. For our experimental investigations, we used $\mathrm{NaCl}$ in deionized water at various weight percentages in order to evaluate the sensitivity. Developing application-specific sensors is typically a balancing act between sensitivity and optical losses traded off against each other within the limitations of the present fabrication flow. On the one hand, narrowing the line width (FWHM) reduces the detection limit. This can be achieved by lowering optical losses within the ring resonator. On the other hand, lower losses are primary observed through strong confinement inside the silicon waveguide, which leads to a lower interaction with the fluid. As a consequence, the ring resonator sensitivity is reduced at the same time.
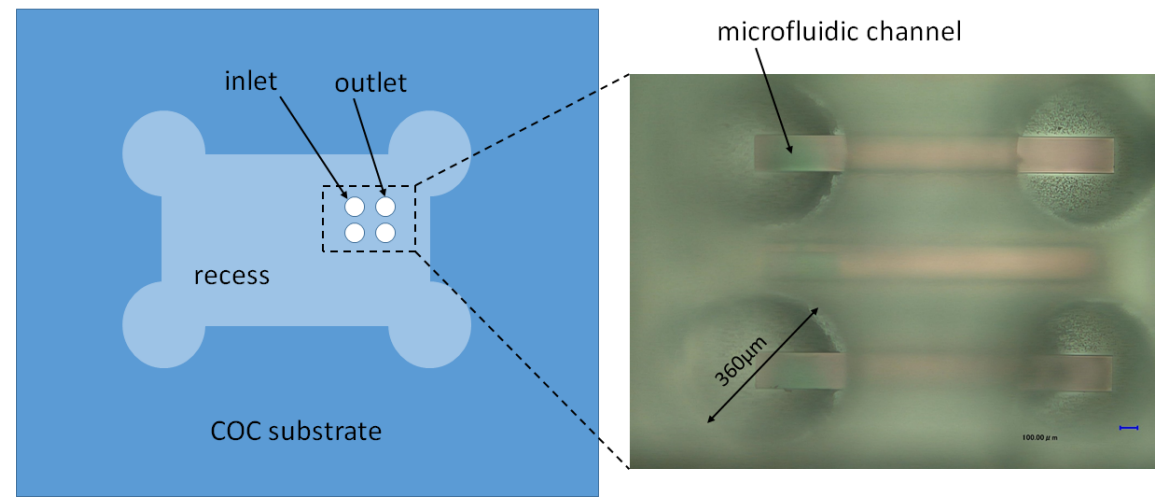

Figure 2. (a) Schematic view of the COC substrate with recess for the sensor-chip. (b) Microscopic picture of the inand outlet as well as the microfluidic channel.

One strategy to find a trade-off is the use of a partially slotted ring resonator. ${ }^{10}$ This approach combines a rib waveguide with a slot waveguide. ${ }^{11}$ The slot waveguide exhibits higher optical losses, which are caused by random line-edge sidewall roughness scattering. However, its high sensitivity makes it suitable for sensing applications. ${ }^{12}$ Here, we use a rib waveguide and a strip-loaded slot waveguide, as it is usually use in siliconorganic hybrid photonics. ${ }^{13,14}$ The strip-loads are usually used for electro-optical modulators ${ }^{15,16}$ but here we use them as etch stop.

As proof on concept, we used $\mathrm{NaCl}$ dispersed in DI water at different weight percentages. The refractive index $n_{f}$ of the $\mathrm{NaCl}$ solved in DI water at a wavelength of $1550 \mathrm{~nm}$ can be calculated by

$$
n_{f}(w t \%)=1.3105+0.17151 \times \frac{C(w t \%)}{100},
$$

where $C(w t \%)$ represents the concentration in weight percentage. ${ }^{17,18}$ We injected four different concentrations ranging from $0 \mathrm{wt} \%$ to $3 \mathrm{wt} \%$. The overall photonic device sensitivity is defined as ${ }^{19}$

$$
S=\frac{\Delta \lambda_{\text {res }}}{\Delta n_{f}},
$$

where $\Delta \lambda_{\text {res }}$ and $\Delta n_{f}$ are the change of the resonance wavelength position and the change of the refractive index of the fluid ( $\mathrm{NaCl}$ in DI water). We revealed a ring resonator sensitivity of about $100 \mathrm{~nm} / \mathrm{RIU}$, which is slightly lower to our previous experiments without microfluidics. ${ }^{20}$ Since the present results are derived from preliminary experiments, further investigations are necessary to verify the sensitivity. However, we have demonstrated the feasibility of a monolithically integrated microchannel for on-chip optofluidic applications. 


\section{CONCLUSIONS}

We have demonstrated the feasibility of monolithically integrated microfluidic channels in conjunction with a COC-based microfluidic interconnection. Micro-milling is used to fabricate the microfluidic interconnection and initial tests showed its feasibility. This work paves the way towards disposable photonic biosensor chips due to its simplicity in terms of microfluidic interconnection and the cost-effective chip area reduction.

\section{ACKNOWLEDGMENTS}

This work was supported in part by the ATTRACT Project funded by European Council (EC) under Grant 777222 and in part by the European Regional Development Fund under Grant 10.13039/501100008530.

\section{REFERENCES}

[1] Luan, E., Shoman, H., Ratner, D. M., Cheung, K. C., and Chrostowski, L., "Silicon photonic biosensors using label-free detection," Sensors 18(10), 3519 (2018).

[2] Mai, A., Bondarenko, S., Mai, C., and Steglich, P., "Photonic thermal sensor integration towards electronicphotonic-ic technologies," in [ESSDERC 2019 - 49th European Solid-State Device Research Conference (ESSDERC)], 254-257 (2019).

[3] Kita, D. M., Michon, J., Johnson, S. G., and Hu, J., "Are slot and sub-wavelength grating waveguides better than strip waveguides for sensing?," Optica 5(9), 1046-1054 (2018).

[4] Molina-Fernández, Í., Leuermann, J., Ortega-Moñux, A., Wangüemert-Pérez, J. G., and Halir, R., "Fundamental limit of detection of photonic biosensors with coherent phase read-out," Optics express 27(9), 12616-12629 (2019).

[5] Rahim, A., Goyvaerts, J., Szelag, B., Fedeli, J., Absil, P., Aalto, T., Harjanne, M., Littlejohns, C., Reed, G., Winzer, G., Lischke, S., Zimmermann, L., Knoll, D., Geuzebroek, D., Leinse, A., Geiselmann, M., Zervas, M., Jans, H., Stassen, A., Domínguez, C., Muñoz, P., Domenech, D., Giesecke, A. L., Lemme, M. C., and Baets, R., "Open-access silicon photonics platforms in europe," IEEE Journal of Selected Topics in Quantum Electronics 25(5), 1-18 (2019).

[6] Bondarenko, S., Hülsemann, M., Mai, A., and Steglich, P., "Fiber-to-chip light coupling using a graded-index lensed fiber collimator," Optical Engineering 60(1), 014105 (2021).

[7] Mai, C., Steglich, P., Fraschke, M., and Mai, A., "Back-side release of slot waveguides for the integration of functional materials in a silicon photonic technology with a full beol," IEEE Transactions on Components, Packaging and Manufacturing Technology 10(9), 1569-1574 (2020).

[8] Mai, C., Steglich, P., and Mai, A., "Adjustment of the beol for back side module integration on wafer level in a silicon photonic technology," in [MikroSystem Technik 2019; Congress], 1-4 (2019).

[9] Steglich, P., Mai, A., and Mai, C., "Photonic sensor chip, packaged photonic sensor device and arrangement," (June 25 2020). US Patent App. 16/718,595.

[10] Steglich, P., Mai, C., Stolarek, D., Lischke, S., Kupijai, S., Villringer, C., Pulwer, S., Heinrich, F., Bauer, J., Meister, S., et al., "Partially slotted silicon ring resonator covered with electro-optical polymer," in [Silicon Photonics and Photonic Integrated Circuits V], 9891, 98910R, International Society for Optics and Photonics (2016).

[11] Steglich, P., Villringer, C., Pulwer, S., Heinrich, F., Bauer, J., Dietzel, B., Mai, C., Mai, A., Casalboni, M., and Schrader, S., "Hybrid-waveguide ring resonator for biochemical sensing," IEEE Sensors Journal 17, 4781-4790 (Aug 2017).

[12] Steglich, P. and You, K., "Silicon-on-insulator slot waveguides: Theory and applications in electro-optics and optical sensing," in [Emerging Waveguide Technology], 187-210, IntechOpen (2018).

[13] Steglich, P., Mai, C., and Mai, A., "Silicon-organic hybrid photonic devices in a photonic integrated circuit technology," ECS Journal of Solid State Science and Technology 8(11), Q217-Q221 (2019).

[14] Steglich, P., Villringer, C., Dietzel, B., Mai, C., Schrader, S., Casalboni, M., and Mai, A., "On-chip dispersion measurement of the quadratic electro-optic effect in nonlinear optical polymers using a photonic integrated circuit technology," IEEE Photonics Journal 11(3) (2019). 
[15] Steglich, P., Villringer, C., Dietzel, B., Mai, C., Schrader, S., Casalboni, M., and Mai, A., "Electric fieldinduced linear electro-optic effect observed in silicon-organic hybrid ring resonator," IEEE Photonics Technology Letters 32(9), 526-529 (2020).

[16] Steglich, P., Mai, C., Villringer, C., and Mai, A., "Direct observation and simultaneous use of linear and quadratic electro-optical effects," Journal of Physics D: Applied Physics 53, 125106 (jan 2020).

[17] Su, H. and Huang, X. G., "Fresnel-reflection-based fiber sensor for on-line measurement of solute concentration in solutions," Sensors and Actuators B: Chemical 126(2), 579-582 (2007).

[18] Tu, X., Song, J., Liow, T.-Y., Park, M. K., Yiying, J. Q., Kee, J. S., Yu, M., and Lo, G.-Q., "Thermal independent silicon-nitride slot waveguide biosensor with high sensitivity," Opt. Express 20, 2640-2648 (Jan 2012).

[19] Steglich, P., Hülsemann, M., Dietzel, B., and Mai, A., "Optical biosensors based on silicon-on-insulator ring resonators: A review," Molecules 24(3), 519 (2019).

[20] Steglich, P., Bondarenko, S., Mai, C., Paul, M., Weller, M. G., and Mai, A., "Cmos-compatible silicon photonic sensor for refractive index sensing using local back-side release," IEEE Photonics Technology Letters 32(19), 1241-1244 (2020). 